\title{
MiR-25 regulates apoptosis by targeting Bim in human ovarian cancer
}

\author{
HAIYAN ZHANG, ZHI ZUO, XIN LU, LI WANG, HAIYAN WANG and ZHILING ZHU \\ Obstetrics and Gynecology Hospital of Fudan University, No. 419, Fangxie Road, Shanghai, P.R. China
}

Received September 6, 2011; Accepted October 13, 2011

DOI: 10.3892/or.2011.1530

\begin{abstract}
MicroRNAs (miRNAs) are emerging as a class of small regulatory RNAs whose alterations are implicated in the initiation and progression of human cancers. Our study showed that miR-25 was highly expressed both in clinical ovarian cancer samples and cell lines. Down-regulation of miR-25 in ovarian cancer cells induced apoptosis whereas overexpression of miR-25 enhanced cell proliferation. The effects of miR-25 abrogation were partly mediated by the intrinsic apoptosis pathway. Many pro-apoptotic proteins such as Bim, Bax and caspase-3 were up-regulated after transfection. Furthermore, luciferase assays demonstrated that Bim was the direct target of miR-25. Introducing Bim cDNA without 3'UTR abrogated miR-25-induced cell survival. Finally, there was an inverse relationship between Bim and miR-25 expression in ovarian cancer tissues. Taken together, these data indicate that miR-25 directly regulates apoptosis by targeting Bim in ovarian cancer and that miR-25 could be a potential therapeutic target for ovarian cancer intervention.
\end{abstract}

\section{Introduction}

MicroRNAs (miRNAs) are groups of non-coding singlestrand RNAs, which modulate protein-coding gene expression post-transcriptionally by interacting with complementary sites within 3'UTR of targets mRNAs (1). They are implicated in a multitude of cellular processes including cell differentiation, proliferation, migration, metabolism, and apoptosis (2-4). Previous studies have pointed out the potential roles of miRNAs in cancers and suggested that abnormal expression of miRNAs may be associated with tumor initiation and progression by regulating cancer-related genes and pathways involved in cancer pathogenesis.

Epithelial ovarian cancer (EOC) is the most common gynecologic malignancy and the sixth most common cancer in women worldwide, with highly aggressive natural history causing almost 125,000 deaths each year (5). Despite advances

Correspondence to: Professor Zhiling Zhu, Department of Gynecology, Obstetrics and Gynecology Hospital of Fudan University, No. 419, Fangxie Road, Shanghai, P.R. China

E-mail: zhilingzhu888@126.com

Key words: ovarian cancer, miR-25, Bim, apoptosis in detection and cytotoxic therapies, only $30 \%$ of patients with advanced-stage ovarian cancer survive 5 years after initial diagnosis (6). Thus, there are urgent needs to develop novel therapeutic approaches by targeting the molecules that are altered in this malignancy. Recent studies showed that miR-25, a member of miR-106b clusters was frequent deregulated in a variety of cancer types (7-9). So far, there are few reports on miR-25 in ovarian cancer.

In our study, we used real-time PCR to analyze the expression of miR-25 both in clinical samples and ovarian cancer cell lines. We certified that miR-25 was high expressed in ovarian cancer. Using a loss-of-function antisense approach, we demonstrated that down-regulation of miR-25 significantly induced apoptosis, while overexpression enhanced proliferation. Meantime, we showed that the effect of miR-25 was partly mediated by targeting the Bim activated intrinsic apoptosis pathway. These results identify a critical role for miR-25 in regulation of proliferation and apoptosis in ovarian cancer, suggesting that miR-25 could be critical therapeutic target for ovarian cancer intervention.

\section{Materials and methods}

Cell lines and culture conditions. Human ovarian cancer cell lines, SKOV3, OVCAR3, OVCAR5, A2780 and ovarian surface epithelial cells, OSE were purchased from Chinese Academy of Sciences Cell Bank. All cells were maintained in a $37^{\circ} \mathrm{C}, 5 \%$ $\mathrm{CO}_{2}$ incubator in DMEM supplemented with $10 \%$ fetal bovine serum (FBS), and routinely passaged at 2- to 3-day intervals. Also, experiments were divided into three groups as blank control group, miRNA scrambled group, and As-miR-25 group.

RNA isolation. Human ovary cancer samples were obtained from the Obstetrics and Gynecology Hospital of Fudan Medical University after informed consent from adult patients diagnosed with ovarian cancer, freshly resected during surgery and immediately frozen in liquid nitrogen for subsequent total RNA extraction. RNA was extracted from tissues and ovarian cancer cell lines and ovarian surface epithelial cells using TRizol reagent (Invitrogen, Carlsbad, USA). Real-time quantification of miRNAs was by stem-loop RT-PCR. For the TaqMan-based real-time reverse transcription-polymerase chain reaction (RT-PCR) assays, the ABI 7300 HT Sequence Detection system (Applied Biosystem, Foster City, CA) was used. All the primers of miR-25, and U6 for TaqMan miRNA 
assays were purchased from GenePharma Co., Ltd. (Shanghai, China). Real-time PCR was performed as described previously (10). The relative gene expression was calculated via a $2^{-\Delta \Delta C t}$ method (11).

Transfection of miR mimics and inhibitors. Synthesized RNA duplexes of miR mimics and miR inhibitors were purchased from GenePharma, Shanghai, China. Cells were transfected with $60 \mathrm{nM}$ of each miR mimic, or inhibitor. Hsa-mir-25 mimics sense: 5'CAUUGCACUUGUCUCGGUCUGA3'. Antisense: 5'AGACCGAGACAAGUGCAAUGUU3'. Hsa-mir-25 inhibitor: UCAGACCGAGACAAGUGCAAUG.

Cell growth assays. The MTT assay was used to determine relative cell growth as follows. SKOV3 and OVCAR3 cells were plated at $10^{4}$ cells per well in 96-well plates with six replicate wells for each condition, transfected with oligonucleotides, and assayed 48-h post-transfection. Cell Growth Assay was performed by MTT (Sigma, St. Louis, MO) as described previously (14). The cell viability was determined at 540-nm absorbance using an enzyme-linked immunosorbent assay plate reader. All data points represent the mean of a minimum of six wells.

Apoptosis assays. SKOV3 and OVCAR3 cells were plated in 6 -well plates and transfected with oligonucleotides. The apoptosis ratio was analyzed 48-h post-transfection using Annexin V FITC Apoptosis Detection kit (BD Biosciences, San Diego, $\mathrm{CA}$ ) according to the manufacturer's instructions. Annexin V FITC and propidium iodide double stain was used to evaluate the percentages of apoptosis.

Luciferase reporter assay. The pGL3-WT-Bim-3'UTRLuc reporter was created by the ligation of Bim 3'UTR PCR products into the $X b a$ I site of the pGL3 control vector (Promega, USA). The pGL3-MUT-Bim-3'UTR-Luc reporter was generated from pGL3-WT-Bim-3'UTR-Luc by replacing the binding site of miR-25 with restriction enzyme cutting site CGGATCCG. For the reporter assay, cells were cultured in 96-well plates and transfected with pGL3-Bim-3'UTR-Luc or pGL3-MUT-Bim-3'UTR-Luc, and As-miR-25. Following a 48-h incubation, luciferase activity was measured using a dual-luciferase reporter system (Promega). Luciferase activity was measured $48 \mathrm{~h}$ after transfection with the Dual-luciferase reporter assay system. The Renilla luciferase activity was utilized as an internal control.

Western blot analysis. SKOV3 and OVCAR3 cells were lysed in $1 \%$ Nonidet P-40 lysis buffer $48 \mathrm{~h}$ following control, scramble or miR-25 inhibitor transfection. SDS-PAGE was performed on $40 \mu \mathrm{g}$ of protein from each sample, gels were transferred to PVDF membranes (Millipore, USA) and incubated with primary antibodies against Bim, Bcl-2 (1:1000 dilution, CST, USA), cleaved-caspase-3 and Bax (1:1000 dilution, Santa Cruz, USA), followed by incubation with an HRP-conjugated secondary antibody (1:1000 dilution, Zymed, San Diego, CA). The specific protein was detected using a Super-Signal protein detection kit (Pierce, USA). After washing with stripping buffer, the membrane was re-probed with antibody against GAPDH (1:1000 dilution, Santa Cruz).

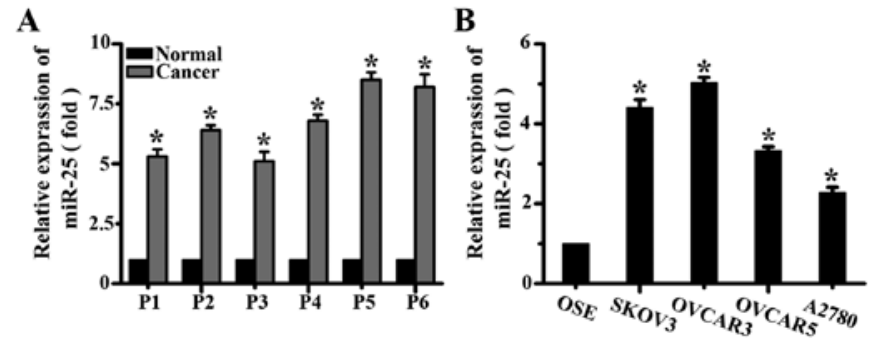

Figure 1. miR-25 shows high expression in ovarian cancer samples and ovarian cancer cells. (A) MiR-25 expression in ovarian cancer and normal ovary tissues by real-time PCR. (B) qRT-PCR analysis showed that SKOV3, OVCAR3, OVCAR5 and A2780 express higher levels of miR-27b compared with ovarian surface epithelial cells, OSE. Data are mean $(+\mathrm{SD})$ of three replicates; ${ }^{*} \mathrm{P}<0.05$.

Statistical analysis. All tests were done using SPSS Graduate Pack 11.0 statistical software (SPSS, Chicago, IL). Descriptive statistics including mean and SE along with one-way ANOVAs were used to determine significant differences. $\mathrm{P}<0.05$ was considered significant.

\section{Results}

MiR-25 is up-regulated in human ovarian cancer samples and cell lines. To investigate the expression of miR-25 in human ovarian cancer samples and cell lines, we performed the TaqMan-based real-time stem-loop RT-PCR analyses. Our data showed that miR-25 was strongly up-regulated in ovarian cancer tissue versus adjacent non-tumor tissue (Fig. 1A). The expression levels of miR-25 in ovarian cancer cell lines were similar with ovarian cancer samples compared with the OSE normal ovarian epithelial cells (Fig. 1B). Taken together, our results demonstrated that miR-25 was highly expressed both in ovarian cancer samples and cell lines.

Down-regulation of $m i R-25$ inhibits proliferation and induces apoptosis in ovarian cancer cell lines. To reveal the effect of miR-25 deletion on cell proliferation, we transfected SKOV3 and OVCAR cells with miR-25 inhibitors and performed MTT assays. First of all, real-time PCR showed that miR-25 inhibitor reduced miR-25 levels by $89 \%$ in SKOV3 cells and $79 \%$ in OVACR3 cells $(\mathrm{P}<0.05)$ (Fig. 2A). MTT assay showed that proliferation was inhibited in the miR-25 abrogation ovarian cancer cells. The suppression was detected 2 days after transfection, and was more apparent on day 3 (Fig. 2B). We also examined effects of miR-25 inhibitor on apoptosis. Inhibition of miR-25 in SKOV3 and OVCAR cells increased apoptosis as confirmed by Annexin V/PI assay. As shown in Fig. 2C, the percentage of apoptotic cells increased from $4.2 \pm 0.7 \%$ in the negative control to $16.5 \pm 0.5 \%$ in SKOV3 cell transfected with miR-25 inhibitor. Similar effects were also observed in As-miR-25 treated OVCAR3 cells.

Bim is a direct target of miR-25. To investigate how miR-25 operates during the progression of ovarian cancer, we searched for the target genes of miR-25. The gene that was predicted by all the three algorithm programs (pictar, targetscan and mirbase targets) was chosen as the candidate target of miR-25. Among them, Bim, a pro-apototic protein that is involved in the 

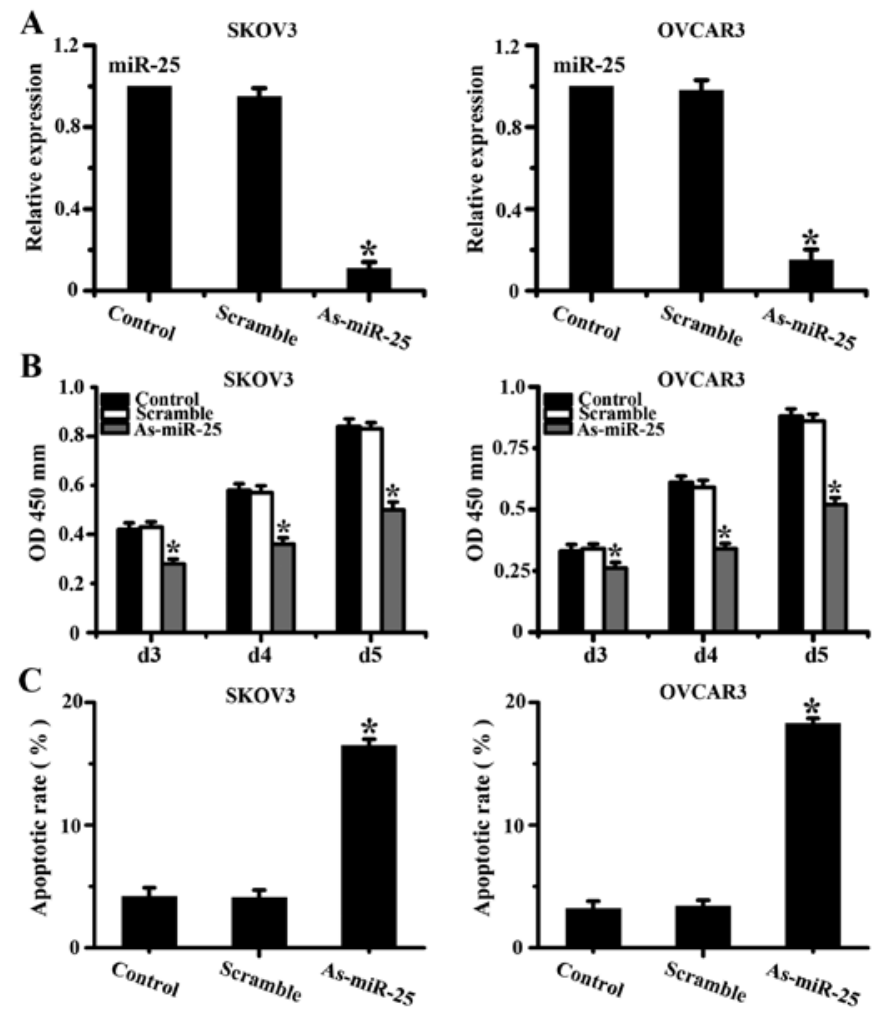

Figure 2. Down-regulation of miR-25 inhibited proliferation and induced apoptosis in ovary cancer cells. (A) miR-25 expression levels (normalized to U6 RNA) were significantly depressed by $85 \%(\mathrm{P}<0.05)$ in SKOV3/ As-miR-25 and $89 \%(\mathrm{P}<0.05)$ in OVCAR3/As-miR-25 cells, relative to the scramble. (B) MTT assay showed that SKOV3/As-miR-25 and OVCAR3/ As-miR-25 cells grew slower than cells transfected with the scramble and control. (C) Apoptosis assay showed that more apoptosis was induced in miR-25 abgroation group compared with control. Data are mean $+\mathrm{SD}, \mathrm{n}=3$; ${ }^{*} \mathrm{P}<0.05$.

intrinsic apoptosis pathway, was found to have a putative miR-25 binding site within its 3'UTR (Fig. 3A). To detect whether Bim is indeed regulated by miR-25, we knocked-down miR-25 in SKOV3 and OVCAR3 cells. Western blot analysis showed that Bim expression was up-regulated in both SKOV3 and OVCAR3 cells after miR-25 down-regulation (Fig. 3B). In addition, we created pGL3-WT-Bim-3'UTR and pGL3-MUT-Bim-3'UTR plasmids. Reporter assays revealed that a reduction of miR-25 triggered a marked increase of luciferase activity from pGL3WT-Bim-3'UTR, but produced no obviously change in the luciferase activity from pGL3-MUT-Bim-3'UTR (Fig. 3C). Taken together, these data indicated that Bim is the direct target of miR-25 at least in ovarian cancer.

Bim restoring overrides $m i R-25$ survival function. Having demonstrated Bim as a direct target of miR-25, we next examined the importance of Bim in miR-25-mediated cell survival. Since A2780 had relatively low expression of miR-25, we transfected Bim lacking 3'UTR into A2780 cell. Real-time PCR and Western blot assay confirmed that Bim could be successfully transfected into A2780 cell (Fig. 4A and B). Proliferation, and apoptosis assay showed that ectopic expression of Bim abrogated miR-25 effects on cell proliferation and apoptosis (Fig. 4C and D). Taken together, these results suggested that Bim is a critical target of miR-25 involved in cell biological behavior.

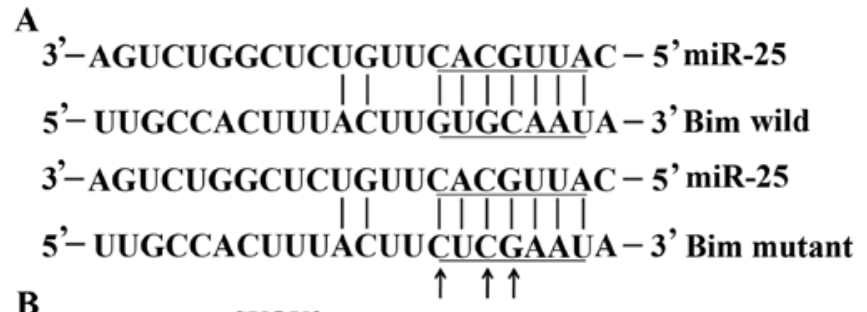

B
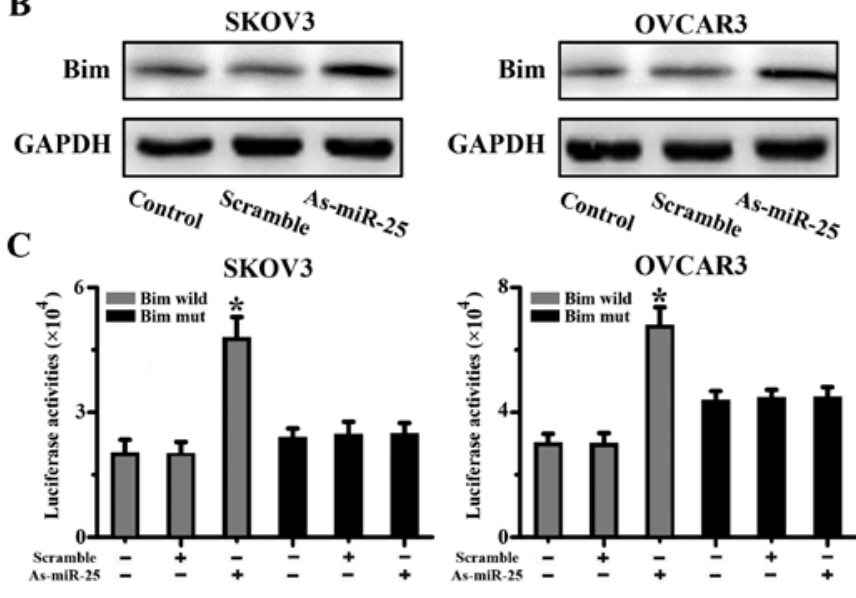

OVCAR3

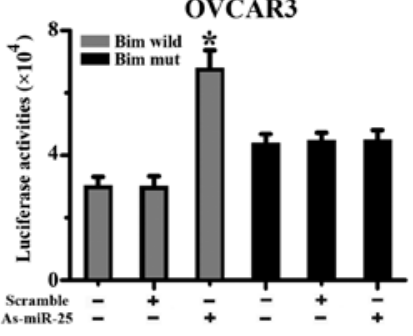

Figure 3. MiR-25 target identification and validation. (A) Bioinformation analysis identified Bim as potential targets for miR-25. Bim wild and mutant luciferase report plasmids were constructed. (B) Western blotting for Bim at 48-h post-transfected of As-miR-25 in SKOV3 and OVCAR3 cells. (C) Luciferase reporter assays confirmed that Bim was the direct target of miR-25 in SKOV3 and OVCAR3 cells, with luciferase activity ranging from 2 - to 3 -fold induction. Each datum represents the mean fold change in expression from three independent experiments $+\mathrm{SE}$.
A
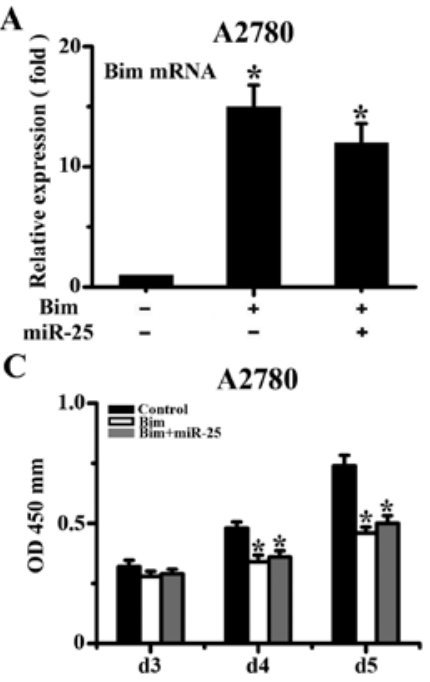

B

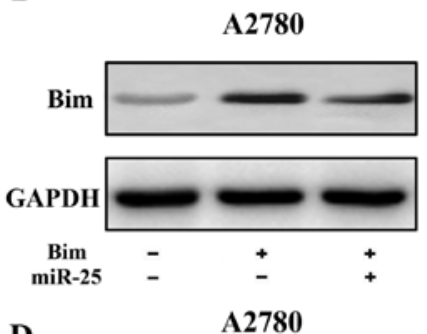

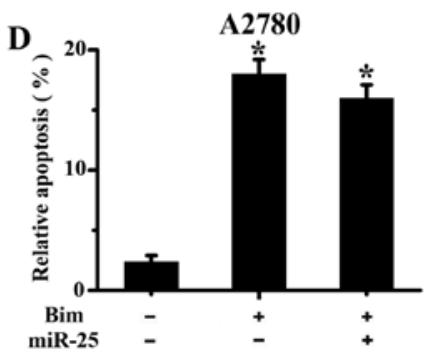

Figure 4. Effects of Bim in ovarian cancer cells. (A) Relative Bim mRNA levels was assessed by qRT-PCR in A2780 cells transfected with expression plasmid of Bim. (B) Bim protein level was assessed by Western blotting in A2780 cells transfected with plasmid of Bim. (C) Proliferation of A2780 cells after Bim transfection with or without miR-25 mimics were significantly reduced compared to control. (D) Significantly increased number of apoptosis was observed in the A498 cells after Bim transfection with or without miR-25 mimics compared to control.

MiR-25 abrogation activates the intrinsic apoptosis pathway. In order to identify some of the further downstream effectors of the observed cellular phenotypic changes, Western 


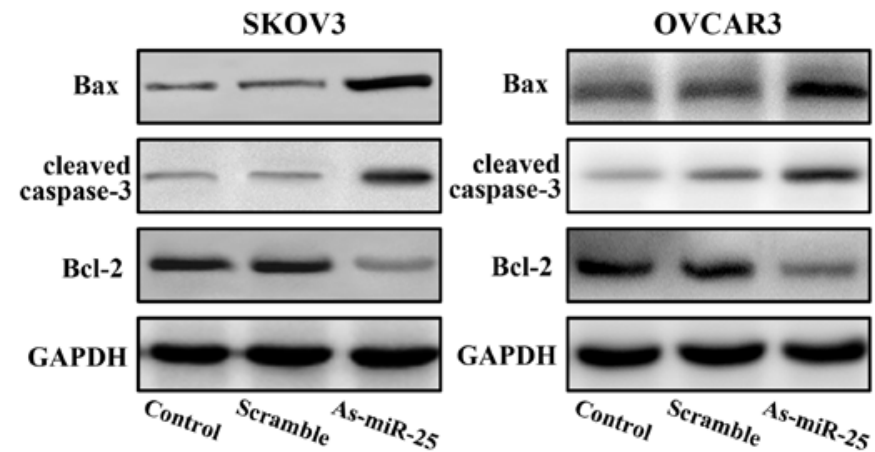

Figure 5. Impact of miR-25 inhibitor on intrinsic apoptosis pathway. SKOV3 and OVCAR3 cells were treated with As-miR-25, as described in Materials and methods. Bax, cleaved-caspase-3, Bcl-2 and GAPDH expression were determined by Western blotting. Data are from one of three representative experiments.

Table I. Clinicopathological parameters of 6 ovarian cancer samples.

\begin{tabular}{lll}
\hline No. & Age & \multicolumn{1}{c}{ Histotype } \\
\hline 1 & 46 & Papillary serous \\
2 & 53 & Endometrioid \\
3 & 61 & Serous \\
4 & 58 & Clear cell \\
5 & 62 & Serous \\
6 & 55 & Clear cell \\
\hline
\end{tabular}

blot assay was evaluated. In two tested ovarian cancer cells, Western blot assay demonstrated that depletion of miR-25 up-regulated the expression of Bax and cleaved-caspase-3, while down-regulated Bcl-2 expression suggested that miR-25 regulated the intrinsic apoptosis pathway via Bax, in ovarian cancer cells (Fig. 5).

Bim is inversely correlated with miR-25 expression in ovary cancer samples. To further evaluate the relationship between miR-25 and Bim in human ovary cancer, we detected the expression of Bim in 6 clinical ovarian cancer samples using Western blot assay. Of the 6 cases, cancer tissues showed decreased Bim expression when compared with adjacent nontumor tissue (Fig. 6A). Associated with Fig. 1, the expression levels of Bim in tumor tissues inversely correlated with the miR-25 levels, suggesting that the decreased Bim expression might result from miR-25 overexpression in human ovarian cancer (Fig. 6B).

\section{Discussion}

In China, ovarian cancer is one of the most frequent invasive malignancies of the female genital tract cancers. Although the majority of ovarian cancer patients will respond to initial chemotherapy, most will ultimately develop disease recurrence (12). To develop more optimized and effective treatment strategies for ovarian cancer, it is critical to gain a deeper understanding of the molecular mechanisms of ovarian cancer
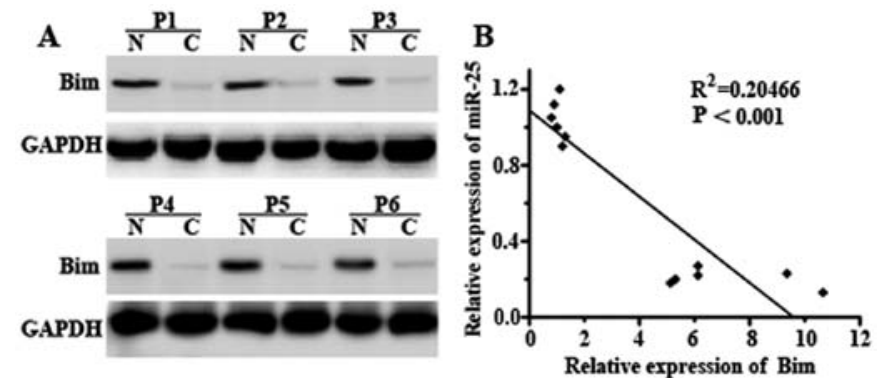

Figure 6. Bim was down-regulated in ovarian cancer samples and inversely correlated with miR-25 levels. (A) The expression of Bim in ovarian cancer samples and normal ovary tissue were assessed by Western blot assay. (B) A statistically significant inverse correlation between miR-25 and Bim protein levels in clinical specimens (Spearman's correlation analysis, R2=0.20466; $\mathrm{P}<0.001)$.

and to identify targets for therapeutic intervention (13-16). MiRNAs have recently been described as important players in human cancer and the role of microRNA as therapeutic targets has been proposed (17-20). So far, there are more than 1000 microRNAs annotated by the latest version of miRBase. The expression of miRNAs is remarkably deregulated in ovarian cancer, strongly suggesting that miRNAs are involved in the initiation and progression of this disease $(21,22)$. In our study, we showed that miR-25 was up-regulated in both ovarian cancer samples and cell lines. Luciferase assay demostrated that the pro-apoptotic protein Bim is a direct target for miR-25.

MiR-25 is a member of MiR-106b cluster which exert potential proliferative, anti-apoptotic, cell cycle-promoting effects in a variety of cancer types (23). Kumar et al used a screening method to identify that miR-25 directly target the 3'UTR of TP53 and reduce apoptosis and cell cycle arrest in lung adenocarcinoma cells (24). Poliseno et al used a computational approach to identify miR-22, miR-25, and miR-302 as three PTEN-targeting microRNA families. MiR-22 and the miR-106b 25 cluster are aberrantly overexpressed in human prostate cancer, correlate with abundance of the miRNA processing enzyme DICER, and potentiate cellular transformation both in vitro and in vivo (7). Furthermore, Kan et al established that miR-106b-25 polycistron is activated by genomic amplification and is potentially involved in esophageal neoplastic progression and proliferation (9). However, there are few reports on miR-25 in ovarian cancer. In this study, we used real-time PCR to confirm that miR-25 was high expressed both in ovarian cancer samples and cell lines. Using sequence-specific antisense knockdown of miR-25, MTT and apoptosis assay indicated that miR-25 abgoration results in growth inhibition and apoptosis induction in ovarian cancer cells.

Bim was first cloned as a Bcl-2-interacting protein using phage expression library screening (25). It contained three major isoforms, BimEL, BimL and BimS produced by alternative splicing. Reportedly, Bim can trigger apoptosis by at least two different mechanisms, by the interaction and neutralization of Bcl2-like molecules and/or direct activation of Bax, and that the decision on which mode of action ensues perhaps depends on the nature of the incoming apoptosis signal. Low expression of Bim has been shown for tumor entities, including melanoma and renal cell carcinoma (26). The mechanism 
suppressing Bim in these cancer types has not been clarified yet. In our study, Western blot assay delineated that Bim had low expression in ovarian cancer samples compared with normal ovary tissues. Mechanistic investigation revealed that down-regulation of miR-25 activated the intrinsic apoptosis pathway via targeting the 3'UTR of Bim mRNA. Western blotting showed that Bax and cleaved-caspase-3 were up-regulated, while Bcl-2 was decreased.

In summary, we demonstrate that down-regulation of miR-25 induces apoptosis of ovarian cancer by repressing expression of Bim. Genetic restoration of Bim bypassed the effects of miR-25 overexpression, activating the intrinsic apoptosis pathway. Based upon these observations, we conclude that miR-25 is a potential therapy target for ovarian cancer.

\section{Acknowledgements}

This study was supported by Shanghai Natural Scientific Fund (09ZR1404700).

\section{References}

1. Bartel DP: MicroRNAs: genomics, biogenesis, mechanism, and function. Cell 116: 281-297, 2004.

2. Kloosterman WP and Plasterk RH: The diverse functions of microRNAs in animal development and disease. Dev Cell 11: 441-450, 2006

3. Meng F, Henson R, Wehbe-Janek H, Ghoshal K, Jacob ST and Patel T: MicroRNA-21 regulates expression of the PTEN tumor suppressor gene in human hepatocellular cancer. Gastroenterology 133: 647-658, 2007.

4. Jovanovic M and Hengartner MO: miRNAs and apoptosis: RNAs to die for. Oncogene 25: 6176-6187, 2006.

5. Cannistra SA: Cancer of the ovary. N Engl J Med 351: 2519-2529, 2004.

6. Greenlee RT, Hill-Harmon MB, Murray T and Thun M: Cancer statistics, 2001. CA Cancer J Clin 51: 15-36, 2001.

7. Poliseno L, Salmena L, Riccardi L, et al: Identification of the miR-106b 25 microRNA cluster as a proto-oncogenic PTENtargeting intron that cooperates with its host gene MCM7 in transformation. Sci Signal 3: ra29, 2010.

8. Li Y, Tan W, Neo TW, Aung MO, Wasser S, Lim SG and Tan TM: Role of the miR-106b-25 microRNA cluster in hepatocellular carcinoma. Cancer Sci 100: 1234-1242, 2009.
9. Kan T, Sato F, Ito T, et al: The miR-106b-25 polycistron, activated by genomic amplification, functions as an oncogene by suppressing p21 and Bim. Gastroenterology 136: 1689-1700, 2009.

10. Chen C, Ridzon D, Broomer AJ, et al: Real-time quantification of microRNAs by stem-loop RT-PCR. Nucleic Acids Res 33: e179, 2005.

11. Livak KJ and Schmittgen TD: Analysis of relative gene expression data using real-time quantitative PCR and the 2(-Delta Delta C(T)) method. Methods 25: 402-408, 2001.

12. Jelovac D and Armstrong DK: Recent progress in the diagnosis and treatment of ovarian cancer. CA Cancer J Clin 61: 183-203, 2011.

13. Dahiya N and Morin PJ: MicroRNAs in ovarian carcinomas. Endocr Relat Cancer 17: F77-F89, 2010.

14. Spannuth WA, Sood AK and Coleman RL: Angiogenesis as a strategic target for ovarian cancer therapy. Nat Clin Pract Oncol 5: 194-204, 2008.

15. Darcy KM and Schilder RJ: Relevant molecular markers and targets. Gynecol Oncol 103: S6-S13, 2006.

16. Tan DS, Agarwal R and Kaye SB: Mechanisms of transcoelomic metastasis in ovarian cancer. Lancet Oncol 7: 925-934, 2006.

17. Nana-Sinkam SP and Croce CM: MicroRNAs as therapeutic targets in cancer. Transl Res 157: 216-225, 2011.

18. Law PT and Wong N: Emerging roles of microRNA in the intracellular signaling networks of hepatocellular carcinoma. J Gastroenterol Hepatol 26: 437-449, 2011.

19. Gandellini P, Profumo V, Folini M and Zaffaroni N: MicroRNAs as new therapeutic targets and tools in cancer. Expert Opin Ther Targets 15: 265-279, 2011.

20. Farazi TA, Spitzer JI, Morozov P and Tuschl T: miRNAs in human cancer. J Pathol 223: 102-115, 2011.

21. Spiliotis J, Halkia E and Roukos DH: Ovarian cancer screening and peritoneal carcinomatosis: standards, 'omics' and miRNAs for personalized management. Expert Rev Mol Diagn 11: 465-467, 2011.

22. Shih KK, Qin LX, Tanner EJ, et al: A microRNA survival signature (MiSS) for advanced ovarian cancer. Gynecol Oncol 121: 444-450, 2011.

23. Kim YK, Yu J, Han TS, et al: Functional links between clustered microRNAs: suppression of cell-cycle inhibitors by microRNA clusters in gastric cancer. Nucleic Acids Res 37: 1672-1681, 2009.

24. Kumar M, Lu Z, Takwi AA, et al: Negative regulation of the tumor suppressor p53 gene by microRNAs. Oncogene 30: 843-853, 2011.

25. O'Connor L, Strasser A, O'Reilly LA, et al: Bim: a novel member of the Bcl-2 family that promotes apoptosis. EMBO J 17: 384-395, 1998.

26. Piñon JD, Labi V, Egle A and Villunger A: Bim and Bmf in tissue homeostasis and malignant disease. Oncogene 27: S41-S52, 2008. 